\title{
Gestión de procesos de comunicación como estrategia de competitividad- calidad en Ciudad Juárez, Chihuahua
}

DOI: $10.22403 /$ UROOMX/TYP8/05

\begin{abstract}
Omar Chacón
Tomás J. Cuevas Contreras

Francisco Arturo Bribiescas Silva Universidad Autónoma de Ciudad Juárez

\section{RESUMEN}

En el presente artículo se examinan los procesos de comunicación en una empresa de fabricación de lámparas automotrices y su relación con la competitividad-calidad. Se consideran el estrés y el flujo de información interna de la organización para evaluar lo que provoca problemas internos de comunicación, que a su vez repercuten en el desempeño de las capacidades y habilidades personales y en la competitividad de los procesos.Asimismo se observan las relaciones internas entre mandos medios frente a los retos estructurales, cuyos elementos son necesarios para la mejora de procesos administrativos y operaciones de manufactura. Mediante el análisis de la comunicación es posible potenciar una estrategia que, de manera acorde con el entorno, optimice sus procesos. Se evaluaron cinco aspectos relativos a la comunicación y se entrevistó a los encargados de los nodos estratégicos de la empresa, capaces de hacer cumplir los requerimientos de sus clientes.
\end{abstract}

Palabras |Comunicación efectiva, competitividad, calidad, procesos

CLAVE operacionales, manufactura esbelta 


\section{Introducción}

A partir de 1970, la competencia en los sectores productivos se desarrolló con gran fuerza y de manera impredecible; para enfrentarla, se plantearon nuevas estrategias operativas dentro del ámbito de la manufactura, que la llevaron a ser más eficiente, rápida y flexible (Prahalad y Hamel, 1990). Entre las innovaciones generadas se encontraban los sistemas de administración estratégica, modelos de control de calidad, nuevas estrategias de manufactura y formas organizacionales más ágiles y flexibles.

Actualmente se dispone de una amplia literatura que explica y analiza las propuestas de modelos conceptuales y teóricos estudiados y validados por diversos autores expertos en estrategias de manufactura (EM), que se aplican en las empresas manufactureras, donde es evidente que hay errores y problemas en la toma de decisiones. Las estadísticas y datos empíricos indican la existencia de problemas, y revelan que se implantan soluciones inefectivas en el área de manufactura.

En la literatura se expone una gran cantidad de casos de análisis y en la industria local abunda la evidencia empírica donde se observan diversos inconvenientes: resistencia al cambio; el personal no acepta ni asimila las metodologías y técnicas correctas, lo cual dificulta la ejecución efectiva; resistencia al aprendizaje, derivada en gran parte de lo anterior; ausencia de estrategias de planeación; educación profesional no acorde a la competitiva situación actual; una cultura laboral no enfocada al mejoramiento; una estructura organizacional demasiado vertical; falta de respeto a la ergonomía dentro de las áreas operacionales; calidad basada en contenciones, la cual resulta de problemas de máquinas, personal mal entrenado y ausencia de mantenimiento del equipo operacional.

\section{Planteamiento del problema}

En la industria automotriz, como en cualquier otra industria que compite por mantener clientes y ganar nuevos, es muy importante el tiempo de respuesta al cliente final en cuanto a solicitudes de cambios de ingeniería, incrementos en la demanda, modificaciones de empaque, etc. En el entorno competitivo de hoy ( $y$ de siempre), una empresa debe ser buena en lo que hace y poseer habilidades que le permitan destacar. Su pronta respuesta hace la diferencia para conseguir nuevos negocios y sobresalir en el ramo sin descuidar la calidad del producto. 
El caso de estudio es la compañía de capital italiano y tecnología alemana: Tecnología de lluminación, que se dedica a la fabricación de lámparas automotrices para clientes como Honda, Ford, General Motors, BMW, Mercedes, Nissan y Hyundai. Los empleados son de diferentes nacionalidades: italianos, alemanes, estadounidenses, franceses, argentinos y brasileños, lo cual, por razones culturales e idiomáticas, complica el flujo de la comunicación entre ellos. Adicionalmente, en el sector de manufactura en Ciudad Juárez, tanto de capital nacional como extranjero, se operan técnicas de alto nivel, en las que es común observar que se implementen diversos tipos de tecnologías a través de procesos relativamente inefectivos (porque son tecnologías adaptadas que no responden al contexto local y menos aún operan con la efectividad esperada), lo cual constituye un problema de planeación e información del ambiente.

En la literatura se exponen problemas típicos y complejos: una cultura laboral con bajo nivel de enfoque hacia el mejoramiento, inadecuadas e insuficientes medidas de planeación estratégica gerencial, falta de modelos de producción, decisiones de no inversión en tecnología de punta e informática, etc., en los que se observa la ausencia de modelos administrativos (Caporello, 1996).

Por otra parte, no existe consenso entre autores y expertos sobre los contenidos teóricos, lo que dificulta la selección de metodologías para formular estrategias de comunicación efectiva.Amoako-Gyampah y Boye (I998) estudiaron las metodologías de pronóstico, planeación y programación de producción, prácticas de trabajo en el piso de producción; prácticas para administración de materiales; estrategias de fabricación, inversión en modernización de tecnologías y adquisición de ventaja competitiva; sin embargo, no hay una práctica industrial generalizada y efectiva para el diseño de estrategias. La falta de vinculación teoría-práctica en las empresas se refleja en sus costos, en la competitividad y en la incorporación o no de tecnología para resolver este tipo de problemas.

Algunos cuestionamientos inherentes a la comunicación y a la competitividad-calidad de la empresa son: ¿cómo coordinar dentro de las estrategias metas y métricos?, ¿cómo trabajar la comunicación estratégica tanto en las operaciones de planta como en el plano corporativo?, ¿cómo utilizar y sacar ventaja de los diferenciales entre lo actual y lo que debería ser adecuado o mejor?, ¿cuáles son los factores que afectan la competitividad del negocio? 
¿Por qué este planteamiento?

En el proceso de flujo de valor de una organización que utiliza EM, en la que los procesos deben ser sensibles a los clientes por medio de la flexibilidad y la agilidad, se crea un enfoque de comunicación. El cliente está unido a esta cadena de flujo de valor por su misma creación de valor, al igual que la integración de proveedores. En la industria local hay organizaciones que están desarrollando EM con bajos resultados operativos de eficiencia, por la falta de información y documentación de las fallas y sus causas; lo que explicaría la práctica común de tomar decisiones sin considerar factores estratégicos y cualitativos dentro del desarrollo de EM.

\section{Objetivo general}

Se pretende mejorar los flujos de comunicación entre departamentos y áreas estratégicas (nodos), como una estrategia para reubicarse en una posición competente y de pronta respuesta entre departamentos, y así optimizar los tiempos de respuesta al cliente final con productividad-calidad.

Objetivos específicos

- Revelar las causales del bajo nivel de comunicación empresa-empleados

- Ampliar los niveles de comunicación entre los departamentos

- Conjugar las capacidades de los recursos humanos, impulsando el conocimiento mediante la comunicación interdepartamental

\section{Relación de contenidos teóricos de las estrategias de manufactura (EM)}

Skinner fue el primero en articular y proponer el concepto de EM para evitar el aislamiento de esta área del resto de las funcionales y de la estrategia de competencias de las firmas. También señala que los elementos más importantes del contenido de la EM pueden ser capturados en dos áreas: prioridades competitivas o capacidades, y categorías de decisión estratégica.

Hayes y Wheelwright (1984) distinguen entre las categorías de decisión de manufactura de una estructura e infraestructura natural, donde las decisiones 
estructurales causan un impacto de largo plazo, son difíciles de revertir, requieren inversión sustancial de capital e incluyen decisiones relacionadas con la capacidad, las conveniencias, la tecnología y la integración vertical. Las decisiones de infraestructura son más tácticas debido a que encierran incontables decisiones y no requieren altas inversiones de capital, y las decisiones que le conciernen son las fuerzas de trabajo, calidad, planeación de producción-control de materiales, organización, desarrollo de procesos de nuevos productos, medición del desempeño y sistemas de compensación.

La EM está caracterizada como la consistencia de un patrón de muchas decisiones individuales que afectan la habilidad de la firma para cumplir los objetivos a largo plazo; en este sentido, su efectividad puede ser medida por su fijación entre las prioridades competitivas.

Voss (1995) defiende la existencia de tres paradigmas de la selección y contenido en EM: a) competir a través de las capacidades, b) las elecciones estratégicas en la EM y c) mejores prácticas de manufactura, que escalan a un desarrollo superior y de capacidad. Pero la revisión de la literatura revela distintos acercamientos o puntos de vista teóricos y escasa investigación empírica en el campo. Los estudios aplicados en firmas de manufactura hacen patente una gran confusión en cuanto a la terminología en este ámbito.

Cabe considerar la influencia de la alineación entre la EM y la estrategia de negocio en función del mismo y la contribución del funcionamiento de la manufactura al funcionamiento del negocio; la estrategia desempeña el papel de acoplamiento entre los requerimientos del mercado externo, la organización interna y los recursos tecnológicos, la capacidad y la ventaja competitiva. Sin una estrategia apropiada, los recursos y las capacidades tecnológicas y de organización estarán dirigidos equivocadamente.

De acuerdo con Sun (2002), las estrategias funcionales pueden incluir la estrategia de manufactura, la de mercado y la de investigación y diseño. La EM es un eslabón entre la estrategia de negocio, la organización y la base tecnológica interna. Al servir como eslabón, la EM es alineada con la estrategia de negocios. Esta alineación o consistencia interna-externa se mira como uno de los paradigmas en la EM (Voss, 1995); sin embargo, no se le ha prestado suficiente atención. Para ganar ventajas competitivas, una firma debe intentar responder con eficacia al ambiente externo (Porter, 1979). No obstante, las estrategias sirven para explotar la capacidad de una corporación como arma competitiva 
para alcanzar su misión y objetivos, tradicionalmente no se ha creído que las actividades de manufactura puedan contribuir a las ventajas competitivas, puesto que son consideradas como operativas y no se basan en la eficacia máxima (Avella, Fernández y Vázquez 1999).

Mediante la revisión de la literatura es posible deducir que la estrategia de manufactura pueda apoyar a la de negocio, y que la de negocio tiene la posibilidad de basarse en la capacidad de la manufactura.

Los datos estadísticos reflejan que las firmas que casi nunca transforman sus estrategias y metas corporativas en una EM tienen una rentabilidad promedio de menos cuatro por ciento, mientras que aquellas que convierten formalmente sus estrategias de negocio en una EM obtienen un aumento promedio de 15 por ciento en su rentabilidad. Por otro lado, hay algunas implicaciones, como la necesidad de un cambio de actitud, para que ésta se alinee con los objetivos de manufactura, los procesos, la tecnología, la organización y los recursos humanos. Además debe incluir la mercadotecnia, la manufactura, la investigación y el diseño. Al formular una estrategia, es necesario desarrollar herramientas o métodos para establecer una perspectiva de funcionamientos múltiples.

Cheng y Musaphir (1996) muestran algunos factores y pasos que se deben tener en cuenta en las estrategias de negocios y en las EM, resaltando la falta del eslabón con la estrategia corporativa. También consideran puntos clave (demostrados en estudios organizacionales), para darle el enfoque estratégico en la implementación, la cultura corporativa, mediciones de desempeño, toma de decisiones y estilos gerenciales. Asimismo, ofrecen tres estudios de organizaciones relacionados con la formulación e implementación de EM; en la identificación de los riesgos y oportunidades, al igual que en el establecimiento de los recursos materiales, técnicos, financieros y administrativos, se encontró una actividad racional conectada. Finalmente, para proveer una ventaja competitiva y apoyar los planes de la alta gerencia, subrayan la visión compartida y un vocabulario común, los cuales unen a la organización y determinan la administración organizacional con los sistemas de medición.

En la implementación de la estrategia se encontró el enfoque del logro de resultados a través de la estructura organizacional, relaciones de procesos y comportamientos, además del liderazgo como un componente clave. En general, se registró que, como un prerrequisito para formular la EM, ésta debe ser reactiva al mercado estratégico desde un análisis externo de competidores y clientes. 
Por su naturaleza, las decisiones de infraestructura son consideradas más tácticas, ya que abarcan incontables decisiones ligadas a aspectos específicos del negocio y, generalmente, no requieren altas inversiones de capital, como fuerza de trabajo, calidad, planeación de producción-control de materiales, organización, desarrollo del proceso de nuevos productos, medición del desempeño y sistemas de recompensa.

Voss (1995) defiende la existencia de los tres diferentes paradigmas para la selección y el contenido de EM:a) compitiendo a través de las capacidades, donde la organización debe alinearse con los factores clave de éxito, su incorporación de estrategias de mercadotecnia y de las demandas de la plaza de mercado; b) las decisiones estratégicas en la EM, basadas en las necesidades internas $y$ externas; y c) las mejores prácticas caracterizadas, por ejemplo, por manufactura de clase mundial, que lidera hacia un desempeño de capacidad superior.

En cuanto a los estudios sobre algunos modelos y marcos de trabajo para examinar cómo las teorías de EM trabajan en la práctica, Demeter (2003) menciona en su tabla comparativa a varios autores y sus teorías, mientras que Meyer y Ferdows (1990) se preguntan si hay conexión entre una EM competitiva y el negocio competitivo, lo cual nos lleva a establecer qué variables o factores son determinantes para su formulación y a revisar el desempeño del negocio en términos de los efectos-resultados en la producción, tales como la calidad y el costo competitivo, según Phillips (1983).

A su vez, se percataron de que no es suficiente la implementación de algunos programas de producción, por lo cual es necesario buscar un portafolio estratégico para un buen desempeño. Autores como Beaumont y Schroeder (1997), mencionados por Demeter (2003), han señalado la conexión entre tecnología y desempeño como factor determinante. Los efectos de una producción estratégica se operacionalizan en una o dos variables de acuerdo con Roth y Miller (1992), quienes enfatizan el rol de los gerentes de producción en la toma de decisiones para el buen desempeño del negocio como centro de enfoque.

\section{Función de los diferentes elementos-variables}

El proceso de formulación e implementación de las EM es complejo y no se entendido bien; para demostrar la factibilidad de su utilización, según Platts et al. (1998), deben ser sometidas a prueba. Por otra parte, ese proceso muestra 
también las dimensiones esenciales para entender el cambio estratégico desde un marco tridimensional que se expone en la figura I. Entre el contexto y el proceso se pretende saber cómo este último puede ser construido a la medida en diferentes situaciones; entre el proceso y el contenido se buscan los resultados y salidas del proceso y cómo éstos deben ser implementados, obviamente persiguiendo la efectividad del proceso.

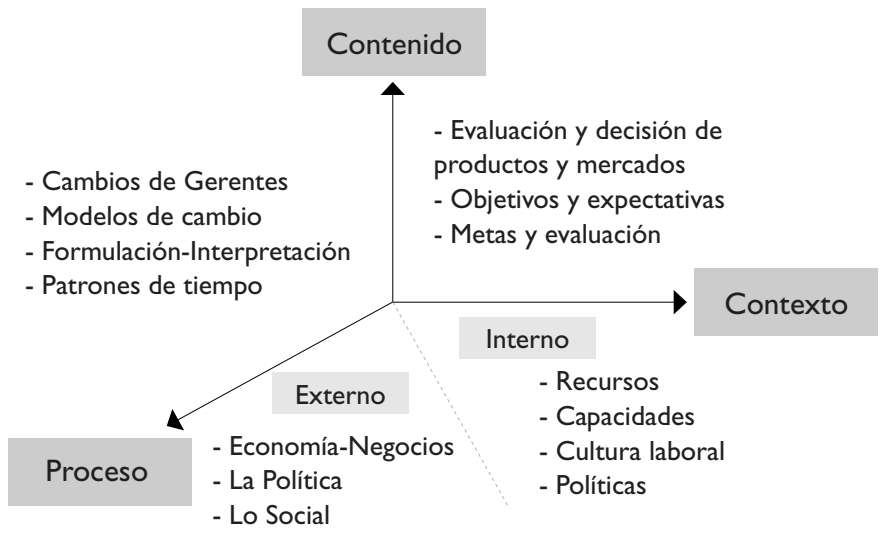

Fuente: Platts et al., 1998

Figura I. Marco tridimensional del cambio estratégico de Platts et al

También, es importante establecer un enfoque para mejorar el entendimiento de cómo el rol de los medidores estratégicos de un sistema de desempeño puede apoyar a las organizaciones a elevar sus estrategias competitivas, alineándolas con el desarrollo organizacional de aprendizaje, como apunta Chenhall (2005), quien, asimismo, señala que las operaciones estratégicas pasan por elementos de la cadena de valor, con un enfoque orientado al cliente y a lo financiero, además del eslabón de proveedores basado en medidores de innovación del negocio, identificando la causa-efecto entre operaciones y la estrategia organizacional.

Así, Dangayach y Deshmukh (2004) muestran un modelo para EM basado en el eslabón que hay entre la competencia de manufactura y el desempeño 
del negocio, además de la previa interrelación de las prioridades competitivas, actividades de mejora y propósito estratégico.

Estas prácticas están estrechamente relacionadas con las prioridades específicas de costo, conformidad de calidad, flexibilidad en volumen y en el diseño, entrega rápida y tiempos de ciclo del producto. Se identifica una alta divergencia dentro de las organizaciones en las metas operacionales, donde constituyen una tarea incierta y deben ser una contingencia clave en la estructura.

La pregunta central en la investigación administrativa es: ¿por qué en algunas organizaciones el desempeño es mejor que en otras? Se ha encontrado que ciertas prácticas y arreglos estructurales están vinculados con el desempeño operacional y financiero, pero aún hay poca evidencia sistemática sobre ello. Un factor limitante es la necesidad de más mediciones directas.

O'Reagan y Ghobadian (2005) estudian el proceso de la innovación en el contexto de estrategia, cultura, estilos gerenciales en innovación eficaz, dentro de la vía rápida y de las EM.Su análisis confirma que las organizaciones de ejecución ponen mayor énfasis en las cualidades de la estrategia, tienen una dirección más fuerte y definida y cultivan los estilos comparados con las firmas de ejecución de punto bajo.

Por otro lado, cabe considerar el impacto de los principales conductores de innovación eficaz: estrategia, dirección y cultura. La investigación sobre la innovación se ha centrado en el costo y los riesgos implicados, donde hay tres importantes factores que conducen al despliegue de la innovación: el plan estratégico de la firma, su capacidad de conducir el proceso y su capacidad de consolidar la innovación. Los recursos humanos de dirección de la cultura del empowerment y las características de la creatividad del personal están asociados a la innovación.

Hipkin (2004) considera la tecnología como una nueva estrategia en un contexto global y muy específicamente en países en desarrollo, desplegando una metodología correlacionada de importancia y control, teniendo en cuenta las barreras tecnológicas entre productos de alto valor y aquellos que valen menos.

\section{Método}

En esta investigación se utilizó el método cualitativo y se aplicaron cuestionarios para determinar el ambiente de trabajo de la empresa Automotive Lighting en las áreas involucradas con el proceso de envíos al cliente, concretamente los 
departamentos considerados "cuellos de botella", a saber, los de Ingeniería, Compras y Producción. Las encuestas confidenciales fueron hechas a los encargados de estas áreas. El procesamiento de los datos se realizó atendiendo más a las cualidades que a las cantidades, con lo que se buscó tener una visión global que estuviera por encima de los detalles. Para recolectar los datos se contó con el Departamento de Logística. Las encuestas se dividieron en cinco partes: I) condiciones de trabajo, 2) cooperación, 3) supervisión, 4) compensaciones, y 5) satisfacción en el trabajo; y se aplicaron a los representantes de 12 áreas consideradas nodos estratégicos del proceso. Se empleó una escala de valoración de uno a cinco, donde uno es la calificación más baja y cinco la más alta, mientras que el cero se tomó como "no se aplica".

\section{Resultados}

En cuanto a las condiciones de trabajo, $75 \%$ del personal se encuentra cómodo en la compañía; $83 \%$ manifiesta que están bien definidas las funciones concernientes a su puesto; y $67 \%$ dice que, al estar capacitado para la labor desempeñada, la compañía le brinda seguridad. Ahora bien, $58 \%$ exterioriza que el trabajo es estresante debido a las actuales condiciones económicas en la manufactura automotriz, por lo que $50 \%$ percibe inseguro el puesto.

A partir de lo anterior, se deduce una adecuada información sobre el puesto y sus tareas, acompañada de los equipos y un ambiente laboral propicio para el desempeño; también se observa nerviosismo por la baja comunicación frente a los acontecimientos financieros globales de la compañía.

En lo que se refiere a la cooperación (gráfica 2), $58 \%$ de los empleados exhibe una tendencia a no importarle lo que los demás departamentos desarrollen; tampoco se sienten involucrados en las actividades empresariales, aunque estén en el mismo departamento. La relación interdepartamental difiere en opiniones (50\%). Esto demuestra diferencias de las metas institucionales entre los empleados y estimula el desinterés por los objetivos generales, alentando la individualidad sobre la unión grupal, debido a la propia integración en su área de trabajo $(75 \%)$.

La lectura del tejido colaborativo y de reciprocidad en el ambiente laboral es preocupante. Se percibe un ambiente de competencia entre departamentos, frente a la falta de información clara de las metas corporativas, lo que, sin duda, 


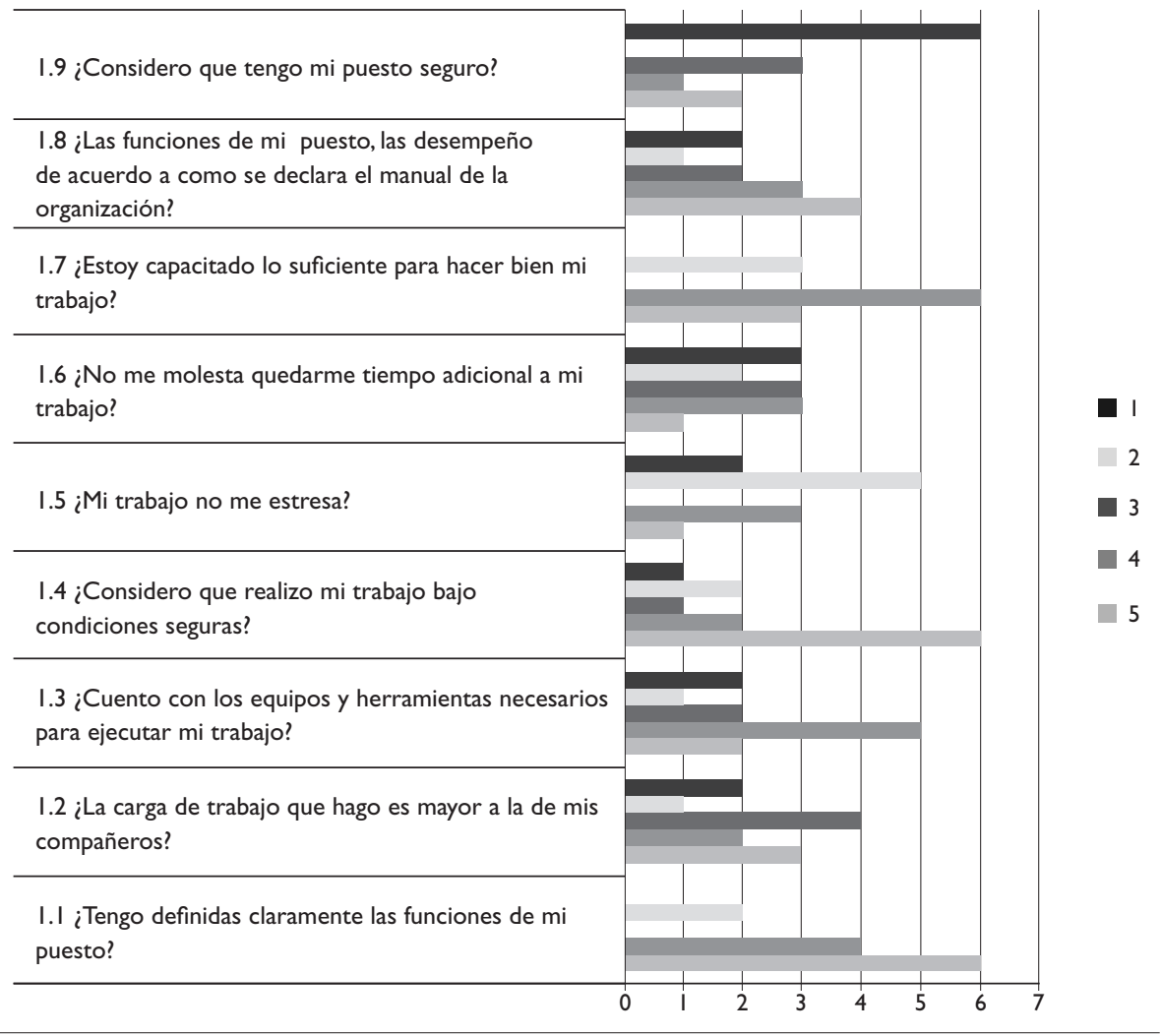

Fuente: Elaboración propia

Gráfica I. Condiciones de trabajo

genera ostracismo y desconfianza en el resto de la organización, provocando, por la falta de comunicación efectiva, resistencia al trabajo en equipo.

Los hallazgos anteriores demuestran las complicaciones de comunicación, manifiestas en el desinterés por cooperar con los departamentos. La cooperación (gráfica 2) se refleja en el apoyo a los supervisores en la empresa (92\%). Se hace patente cómo los entrevistados distinguen el respeto por el supervisor inmediato (83\%) y sienten su apoyo en situaciones comprometidas (92\%); a su vez, sale a relucir el supervisor al exigir lo rigurosamente necesario 
Gestión de procesos de comunicación como estrategia de competitividadcalidad en Ciudad Juárez, Chihuahua

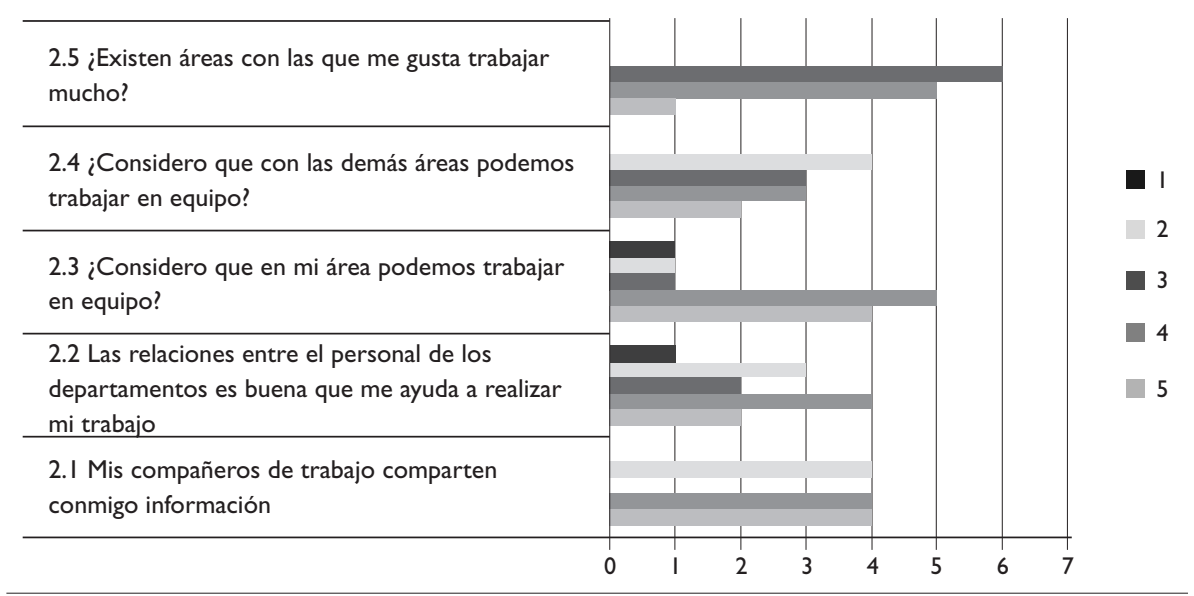

Fuente: Elaboración propia

GRÁFICA 2. CoOperación

( $100 \%)$. Con relación a conflictos de supervisión, no existen problemas entre el supervisor y los subordinados ( $100 \%$ ). Sin embargo, el supervisor es el canal formal de comunicación empresa-empleado que hace realidad el apoyo y el reconocimiento público al buen desempeño, inculca el valor por el trabajo, apoya a la reflexión de los subordinados para la resolución de problemas, ofrece oportunidades de asumir mayores responsabilidades y liderazgo, y confiere los medios para el desarrollo y mejoramiento individual.

Se advierte un alto compromiso en los jefes y supervisores de departamento, mas no en el resto de la estructura de la organización, lo cual es un indicativo de liderazgo basado en la persona, por la falta de directrices claras de la compañía para sus demás empleados. La inexactitud de las políticas de comunicación conlleva cotos de poder, limitando la integración y, por tanto, disminuyendo la capacidad competitivad-calidad.

En lo concerniente a las compensaciones (gráfica 4), la mayoría de los entrevistados no está de acuerdo con las prestaciones recibidas (92\%); discrepan de las evaluaciones hechas por la empresa, las consideran injustas (92\%). Otros (42\%) perciben las promociones como ilegítimas, porque piensan que las reciben quienes no las merecen, pero en parte están de acuerdo con el sueldo 


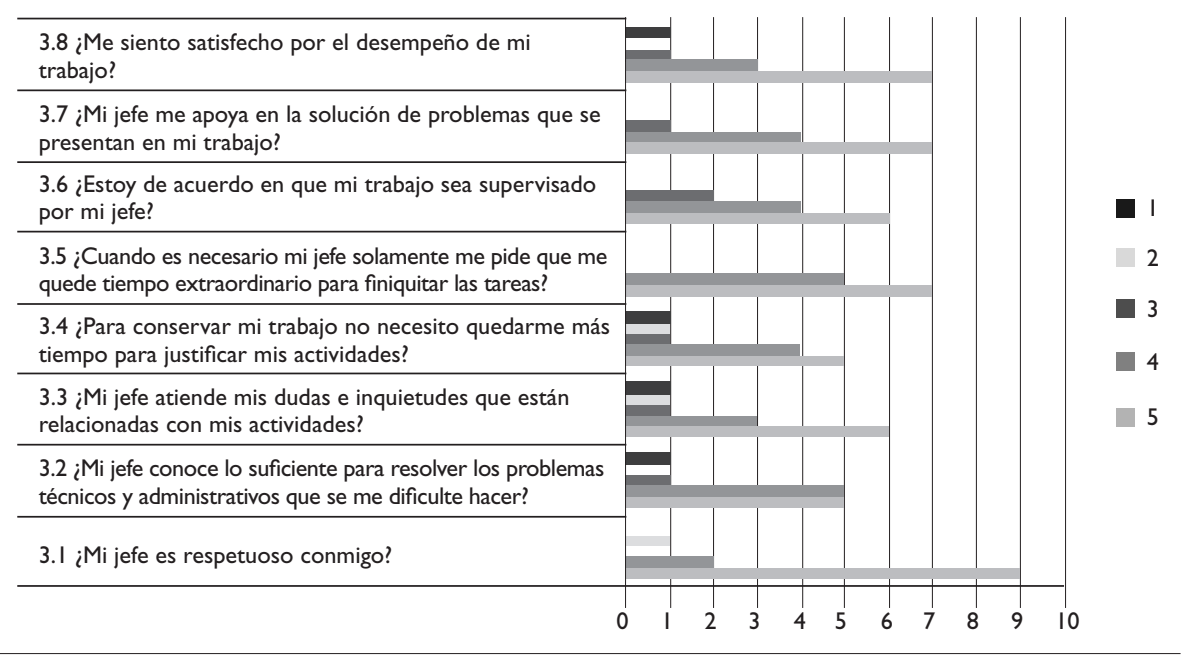

Fuente: Elaboración propia

GRÁFICA 3. SUPERVISIÓN

que perciben, pues en posiciones similares en otras compañías es parecido (58\%). En general, la remuneración por el desempeño es adecuada, aunque es un tópico escabroso en el imaginario del personal y su conformidad con lo que percibe. Empero, es deseable un sistema de recompensas por ideas o proyectos innovadores que permitan a la compañía captar clientes y mejorar su rentabilidad o, en su caso, ahorrar costos. El mensaje es elocuente: se requiere evaluar el área de recursos humanos y sus políticas corporativas, ya que ello ofrece la oportunidad de mejora en las señales subliminales, "me importas, empleado", mediante programas de estímulos al desempeño.

La concordancia de los índices antes expuestos se refleja en la satisfacción en el trabajo que muestra el colectivo, que está conforme con su puesto, su horario $(83 \%)$ y su adecuada relación con sus supervisores $(75 \%)$, al tener buen trato con su jefe inmediato. Asimismo $58 \%$ exterioriza su deseo por permanecer en el departamento actual; no obstante, destaca que $42 \%$ está descontento con la administración general, por no encontrar un adecuado reconocimiento a su desempeño, lo que se traduce en una baja motivación para la consecución de las metas institucionales. 
Gestión de procesos de comunicación

como estrategia de competitividad-

calidad en Ciudad Juárez, Chihuahua

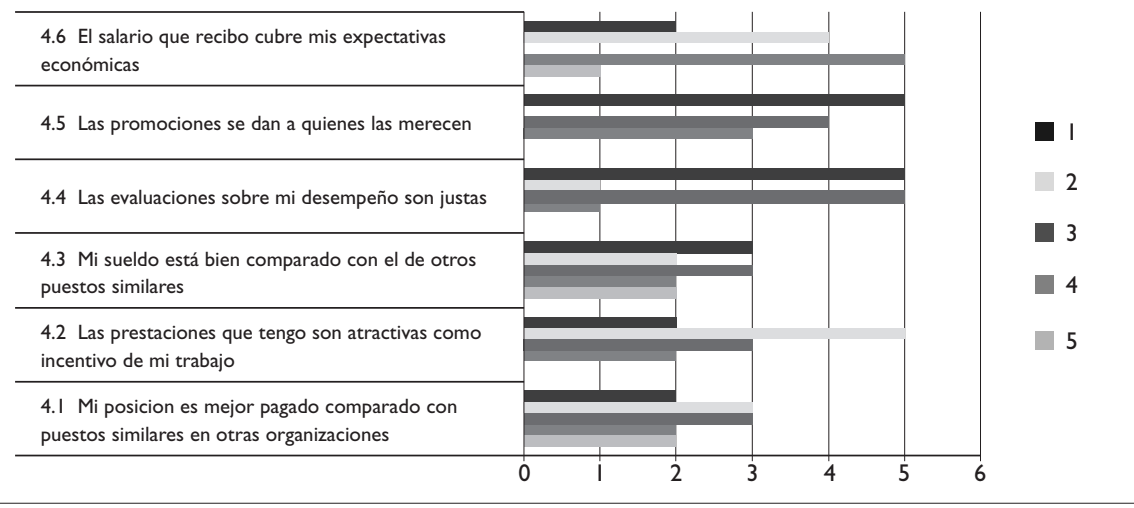

Fuente: Elaboración propia

Gráfica 4. Compensaciones

5.6 Estoy motivado por el reconocimiento que mi jefe y los directivos dan a mi trabajo

5.4 Me gustaría permanecer en mi departamento

5.3 No me iría a otro trabajo aunque me ofrecieran un sueldo similar

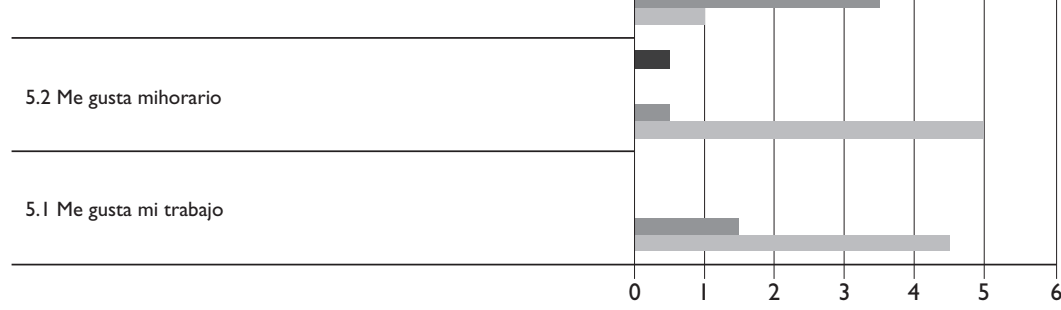

Fuente: Elaboración propia

Gráfica 5. Satisfacción en el trabajo 
Se evidencian los espacios de poder en los departamentos y áreas de trabajo por parte de los jefes y supervisores, aunque la comunicación con la dirección de la empresa esté condicionada por las políticas corporativas de recursos humanos o por la insensibilidad ante sus subordinados.

\section{Conclusiones}

La compañía estudiada requiere prestar mayor atención a la colaboración y cooperación de los empleados, tema central de la encuesta. Aunque ellos se sienten cómodos en su trabajo, la forma en que se distribuye la información de los directivos hacia la corporación no es la más adecuada. La empresa debe procurar que los empleados se sientan integrados a las metas y objetivos corporativos. Es recomendable observar los casos exitosos de comunicación interna, cuyo reflejo son las ventas, y en los que para informar utilizan tableros de comunicación, donde se detallan los pormenores del día. Otras formas de comunicación son adoptar un programa de lean manufacturing, convivir con los proveedores y clientes un día al año, dar a conocer las metas del siguiente año, así como esquemas de comunicación mensuales, con el propósito de informar las condiciones de la empresa y los horizontes deseados.

Para favorecer la alta competitividad se requiere considerar el comportamiento grupal, que redunda en el trabajo en equipo, necesario para la consecución de metas. En cuanto al supervisor, es determinante en la comunicación y motivación del personal; por tanto, ayudarlo a realizar sus tareas de manera responsable y eficiente inspira en sus subordinados buscar objetivos más ambiciosos, aunque carezca del poder para otorgar incentivos financieros.

\section{Recomendaciones}

Se recomienda que en una segunda etapa se obtenga retroalimentación del consumidor final, al estimar los tiempos de entrega y respuesta de los requerimientos, de modo que permita evaluar los procesos y decidir si realmente se llevaron a cabo cambios significativos mediante la flexibilización de la comunicación. 


\section{FUENTES CONSULTADAS}

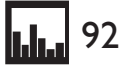

Amoako-Gyampah, K.y S.S. Boye (1998). "Manufacturing Planning and Control Practices and their Internal Correlates:A Study of Firms in Ghana". International Journal of Production Economics, 54, I43-16I.

Avella, L., E. Fernández y C.J. Vázquez (1999). "The Large Spanish Industrial Company:Strategies of the most Competitive Factories”. Omega.The International Journal of Management Science, 27, 497-5I4.

Caporello, T. (1999). "Staying ahead in Manufacturing \& Technology - The Development of Automation Cost of Ownership Model and Examples", en IEEE, International Symposium on Semiconductor Manufacturing Conference Proceedings. Santa Clara: IEEE, 35-38.

Cheng, T.C.E. y H. Musaphir (1996). "Theory and Practice of Manufacturing Strategy”. International Journal of Production Research, 34 (5), I 243-I 259.

Chenhall, R. (2005). "Integrative Strategic Performance Measurement Systems, Strategic Alignment of Manufacturing, Learning and Strategic Outcomes: An Exploratory Study". Accounting, Organizations and Society, 30, 395-422.

Çil, I.y R. Evren (1998).“Linking of Manufacturing Strategy, Market Requirements and Manufacturing Attributes in Technology Choice:An Expert System Approach”. The Engineering Economist, 43 (3), I 83-202.

Cohen, L. y L. Manion (1990). Métodos de investigación educativa. Madrid: La Muralla.

Dangayach, G.S. y S.G. Deshmukh (200I). "Implementation of Manufacturing Strategy: A Select Study of Indian Process Companies”. Production Planning and Control, I2 (I), 89-I05.

Demeter, K. (2003).“Manufacturing Strategy and Competitiveness”. International Journal of Production Economics, 8I-82, 205-2I 3.

Dirube Mañueco, J.L. (2006). "La comunicación interna, aliada estratégica de la gestión empresarial de Novartis”. Capital Humano: Revista para la Integración y Desarrollo de los Recursos Humanos, 19 (202), 62-65.

Hamblin, D. y A. lyer (1996). "What Difference does your Industry Make?”. International Journal of Production Economics, 43 (2-3), 155- 174.

Hayes, R.y S.C.Wheelwright (1984). Restoring our Competitive Edge. Nueva York: John Wiley and Sons.

Hill,T. ( 1 993). Manufacturing Strategy:The Strategic Management of the Manufacturing Function. Londres: Macmillan. 
Hipkin, I. (2004). “Determining Technology Strategy in Developing Countries”. Omega. The International Journal of Management Science, 32, 245-260. Hongyi, S. y C. Hong (2002). "The Alignment between Manufacturing and Business Strategies: Its Influence on Business Performance". Technovation, 22, 699-705.

O'Reagan, N.yA. Ghobadian (2005).“Fast Tracking Innovation in Manufacturing SMEs". Technovation, I-I I.

Platts, K.W., et al. (1998). "Testing Manufacturing Strategy Formulation Processes". International Journal of Production Economics, 56-57, 5I 7-523.

Porter, M.E. (1979). "How Competitive Forces Shape Strategies”. Harvard Business Review, 57 (2), I37-I45.

Prahalad, C.K.y G. Hamel (1990).“The Core Competence of the Corporation”. Harvard Business Review, 68 (3), 79-93.

Voss, C.A. (1995). “Alternative Paradigms for Manufacturing Strategy”. International Journal of Operations and Production Management, I 5 (4), 5- I6.

Voss, C.A.y K. Blackmon (1998). “Differences in Manufacturing Strategy Decisions between Japanese and Western Manufacturing Plants:The Role of Strategic Time Orientation”.Journal of Operations Management, 16 (2), I47-I58.

Ward, P., D. Bickford y G. Keong (1996). "Configurations of Manufacturing Strategy, Business Strategy, Environment and Structure". Journal of Management, 22 (4), 597-626. 